\title{
EVALUACIÓN DE LA REACTIVIDAD DE SULFUROS DE HIERRO Y RESIDUOS MINEROS: UNA METODOLOGÍA BASADA EN LA APLICACIÓN DE LA VOLTAMPEROMETRÍA CÍCLICA
}

\author{
Roel Cruz y Marcos Monroy \\ Instituto de Metalurgia, Facultad de Ingeniería, Universidad Autónoma de San Luis Potosí, Av. Sierra Leona No. 550, \\ Col. Lomas $2^{a}$ Sección, 78210 San Luis Potosí, S.L.P., Mexico \\ Ignacio González* \\ Depto. de Química, Universidad Autónoma Metropolitana, Iztapalapa, Apdo. Postal 55-534, 09340 México D.F., México
}

Recebido em 13/1/05; aceito em 27/7/05; publicado na web em 16/2/06

\begin{abstract}
EVALUATION OF THE REACTIVITY OF IRON SULFIDES AND MINING WASTES: METHODOLOGY BASED ON CYCLIC VOLTAMMETRY. The mining industry around the world produces an important amount of wastes, which by their high toxic metal and iron sulfide content present a serious environmental problem. Iron sulfide oxidation under weathering conditions provokes the main environmental problem of the mining industry, the generation of Acid Rock Drainage (ARD). Up to now the prediction methodologies do not allow the study of important factors that influence the generation of ARD, producing in some cases erroneous or uncertain conclusions. This paper shows the utilization of cyclic voltammetry using carbon paste electrodes (CPE-Mineral) as an alternative tool in the study of the oxidation capacity of iron sulfides and mining wastes. This electrochemical technique constitutes a novel methodology to establish and understand the factors involved during generation of ARD. Results of several studies including selected sulfide samples and sulfide mining wastes have been described in order to show the capacity of this methodology as a complementary tool in the prediction of the generation of ARD.
\end{abstract}

Keyword: sulfide reactivity; acid rock drainage; voltammetry.

\section{INTRODUCCIÓN}

El drenaje ácido de roca (DAR) es el término utilizado para describir el drenaje generado por la oxidación natural de sulfuros minerales contenidos en rocas o productos minerales que son expuestos, a la acción combinada de agua y oxígeno atmosféri$\mathrm{co}^{1}$. Este fenómeno se caracteriza por la generación de efluentes ácidos con alto contenido de iones sulfato y metales pesados en solución, los cuales pueden contaminar aguas superficiales y mantos freáticos ${ }^{1}$. Por lo anterior y considerando que una mina puede llegar a generar anualmente hasta 1,000,000 de toneladas de residuos sulfurosos, se considera al DAR uno de los principales problemas ambientales que enfrenta la industria minera dedicada a la extracción de metales base. La capacidad de los residuos mineros para generar DAR depende, además de su composición mineralógica, de la combinación de diversos factores, tales como la interacción entre los minerales, la formación de productos y subproductos de oxidación, la variación en la composición de los residuos mineros, entre otros ${ }^{1,2}$.

Los minerales responsables de la generación de DAR son los sulfuros de hierro (pirita, $\mathrm{FeS}_{2}$ y pirrotita, $\mathrm{Fe}_{1-\mathrm{x}} \mathrm{S}$ ), los cuales son minerales estables e insolubles mientras no se encuentren en contacto con agua y oxígeno atmosférico. Sin embargo, como consecuencia de la actividad minera, estos dos sulfuros son expuestos a condiciones ambientales oxidantes. El mecanismo de generación de DAR a partir de la oxidación de la pirita ha sido estudiado ampliamente, proponiéndose que las reacciones involucradas $\operatorname{son}^{1,2}$ :

$\mathrm{FeS}_{2}+7 / 2 \mathrm{O}_{2}+\mathrm{H}_{2} \mathrm{O} \rightarrow \mathrm{Fe}^{2+}+2 \mathrm{SO}_{4}^{2-}+2 \mathrm{H}^{+}$

$$
\begin{aligned}
& \mathrm{Fe}^{2+}+1 / 4 \mathrm{O}_{2}+\mathrm{H}^{+} \rightarrow \mathrm{Fe}^{3+}+1 / 2 \mathrm{H}_{2} \mathrm{O} \\
& \mathrm{Fe}^{3+}+3 \mathrm{H}_{2} \mathrm{O} \rightarrow \mathrm{Fe}(\mathrm{OH})_{3}+3 \mathrm{H}^{+} \\
& \mathrm{FeS}_{2}+14 \mathrm{Fe}^{3+}+8 \mathrm{H}_{2} \mathrm{O} \rightarrow 15 \mathrm{Fe}^{2+}+2 \mathrm{SO}_{4}^{2-}+16 \mathrm{H}^{+}
\end{aligned}
$$

El carácter autocatalítico que presenta el mecanismo de generación de DAR, determinado por la oxidación química con ion férrico y la oxidación biológica con bacterias Thiobacillus, provoca que una vez iniciado este fenómeno, sea muy difícil controlarlo sin que esto represente un costo elevado ${ }^{3}$. Por lo anterior, se requiere de técnicas predictivas eficientes para poder proponer medidas de prevención y control del DAR.

En comparación con la pirita, los estudios sobre los mecanismos de disolución de la pirrotita $\left(\mathrm{Fe}_{1-\mathrm{x}} \mathrm{S}\right)$ son escasos ${ }^{4-6}$. En dichos estudios se reporta la cinética de oxidación del mineral en función de características tales como su estructura cristalina, área superficial y el contenido de impurezas en la red cristalina del mineral. Por otra parte, se ha observado que la pirrotita reacciona rápidamente para formar capas superficiales de oxi-hidróxidos de hierro y de sulfuros deficientes de metal ${ }^{5-7}$. Por tal razón, el enfoque de las investigaciones ha sido dirigido a caracterizar la naturaleza de estas especies superficiales con el fin de establecer un mecanismo de reacción de pirrotita ${ }^{6-8}$. En estos estudios se emplean técnicas como XPS, AES, FTIR, Mössbauer y han proporcionado información importante de las especies superficiales formadas durante la oxidación de pirrotita. Sin embargo, no se presenta un análisis del efecto de estas especies en la evolución de la reactividad del mineral en condiciones ambientales. Lo anterior se señala en base a las condiciones y tiempo de alteración a las cuales fue sometida la pirrotita en estos estudios.

El procedimiento típico de predicción de DAR comprende la aplicación de pruebas estáticas y pruebas cinéticas a muestras de 
residuos sulfurosos, procedentes del sitio minero en estudio ${ }^{9}$. Las pruebas estáticas consisten en un balance del contenido de minerales productores de acidez y minerales neutralizadores de acidez, para determinar la capacidad de la muestra para generar DAR. Las muestras potencialmente generadoras de DAR según las pruebas estáticas, son sometidas a las pruebas cinéticas, en las cuales se da seguimiento de la acidez generada y la disolución de iones metálicos a partir de residuos mineros sometidos a una alteración ambiental simulada. Los resultados obtenidos son extrapolados a largos tiempos de alteración, utilizando modelos matemáticos que predicen la generación del DAR y la calidad química del efluente ácido contaminante. Aunque este procedimiento ha sido empleado con éxito, su principal limitación es que sólo da información de los productos finales de la oxidación, mientras que no son evaluados factores tan importantes como la evolución del estado superficial de los sulfuros, los cambios en la composición de las muestras, la interacción entre los minerales presentes, y el efecto de todo ello en la reactividad del mineral. Por tal razón, se requieren metodologías que permitan caracterizar la reactividad de los sulfuros de hierro considerando los efectos de la evolución en su estado superficial, su composición mineralógica y la ocurrencia de impurezas con las que se encuentran asociados en los residuos mineros.

En este trabajo se presenta una metodología basada en el empleo de la técnica de voltamperometría cíclica utilizando electrodos de pasta de carbón, en combinación con las técnicas convencionales de caracterización mineralógica, con el fin de caracterizar la reactividad de pirita, pirrotita y de un concentrado piritoso, procedentes de distintos sitios mineros. La reactividad de estos sulfuros será evaluada en función de su composición mineralógica, del tipo de sulfuros minerales con los cuales se encuentran asociados y de los cambios que se presentan en su estado superficial cuando se someten a condiciones simuladas de intemperismo. Con los resultados obtenidos en este estudio, se espera demostrar la capacidad de la voltamperometría cíclica en el análisis de los procesos que ocurren durante la oxidación de los sulfuros de hierro en los residuos mineros, la cual podría ser utilizada como herramienta complementaria en la predicción del DAR por medio de las pruebas cinéticas.

\section{PARTE EXPERIMENTAL}

\section{Minerales}

Se analizaron 5 muestras de pirita, una de pirrotita y un concentrado piritoso. Las muestras de mineral fueron obtenidas de sitios mineros localizados en Canadá (Huckleberry, Louvicourt y Brunswick) y México (Tizapa y Zimapán). La muestra de Louvicourt generó dos muestras de pirita (Louvicourt 1 y Louvicourt 2) ya que en este sitio se identificaron dos diferentes mineralizaciones piritosas. La muestra de Huckleberry consistió en cristales grandes, lo cual permitió una selección manual de cristales de pirita libres de inclusiones de otros minerales. Las muestras de Tizapa y Louvicourt fueron muestras masivas de grano fino de pirita por lo que no fue posible la separación manual de los minerales no sulfurosos (ganga). La muestra de Brunswick consistió en un concentrado piritoso con partículas de tamaño muy fino $(50 \mu \mathrm{m})$. En esta muestra tampoco fue posible separar los otros minerales que constituyen la muestra. La muestra de pirrotita fue obtenida en la mina El Monte en Zimapán, Hidalgo.

La muestra de pirita de Zimapán proviene de un depósito tipo skarn de plomo-cinc, donde la composición y el intercrecimiento entre los cristales varía debido a la naturaleza de este tipo de depósito y al remplazamiento de minerales formados inicialmente, por minerales formados en la última etapa de la agregación mineralógica ${ }^{10}$. En esta muestra galena $(\mathrm{PbS})$ y esfalerita $(\mathrm{ZnS})$ ocurren como cúmulos masivos o como inclusiones diminutas $(<20$ $\mu \mathrm{m}$ ) dentro de la pirita (Figura 1). Las muestras de Louvicourt, Tizapa y Brunswick provienen de depósitos de sulfuros masivos vulcanogénicos. En este tipo de depósitos, la textura característica del mineral es del tipo mosaico equigranular, donde los sulfuros de plomo y cinc ocurren como parches intersticiales entre los cristales de pirita $^{11,12}$ (Figura 1).

Cada muestra fue lavada por separado en una solución de $\mathrm{HCl}$ $2 \mathrm{~N}$, durante una hora para eliminar todas las posibles capas de
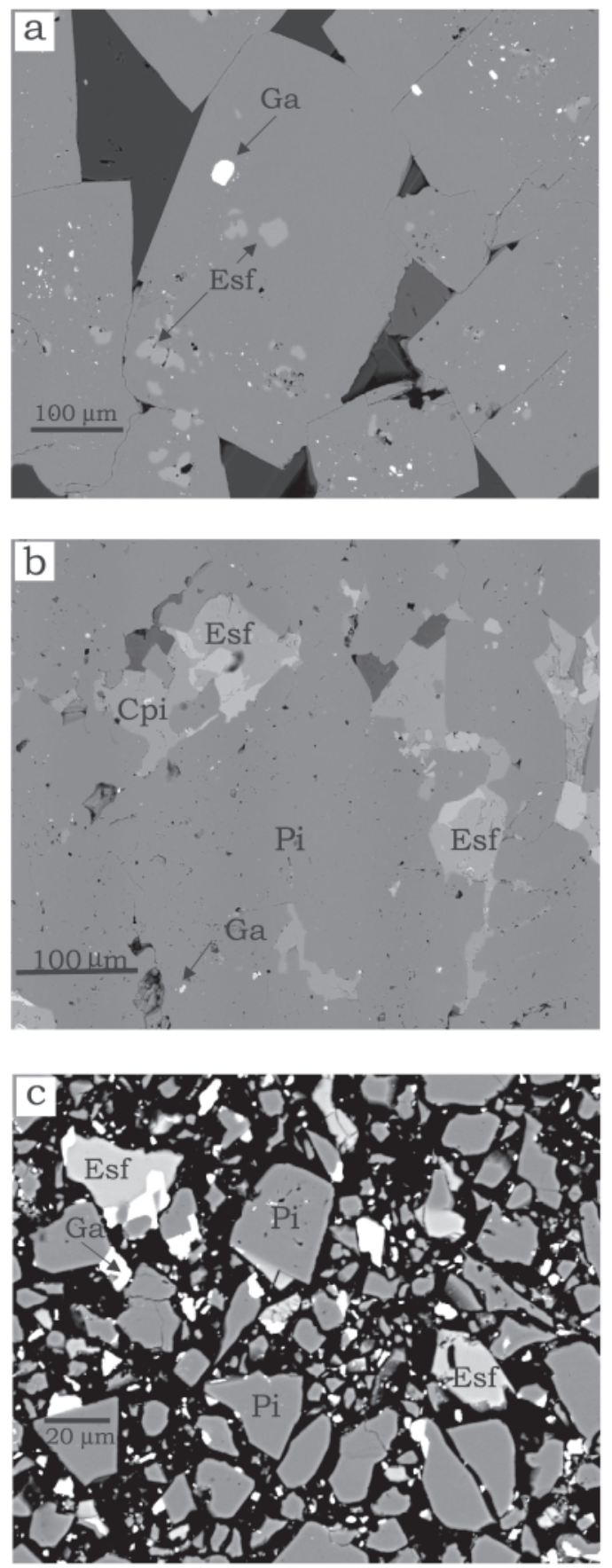

Figura 1. Imágenes de MEB para muestras pulidas: (a) Zimapán: galena (Ga) y esfalerita (Esf) ocurren principalmente como inclusiones en los cristales de pirita, (b) Louvicourt 2: los sulfuros metálicos (galena, Ga; esfalerita, Esf; calcopirita, Cpi) ocurren como parches intersticiales entre los cristales de pirita (Pi), y (c) Brunswick: los sulfuros metálicos están en su mayoría liberados 
óxido presentes en la superficie del mineral. Posteriormente se lavaron con agua desionizada y se secaron a $40{ }^{\circ} \mathrm{C}$ durante $24 \mathrm{~h}$. Todas las muestras, con excepción de Brunswick, fueron fragmentadas en seco utilizando un mortero de ágata para obtener partículas con tamaño de entre 105 a $150 \mu \mathrm{m}$. Las muestras fueron conservadas en una atmósfera de nitrógeno dentro de un desecador hasta su análisis.

\section{Caracterización mineralógica}

Con el fin de determinar la estequiometría de la pirita, su ocurrencia cristalina y el contenido de impurezas mineralógicas, las muestras fueron caracterizadas química y mineralógicamente, antes de ser sometidas a un proceso de alteración que simula el proceso de generación de DAR. Las observaciones mineralógicas fueron realizadas utilizando un microscopio óptico polarizante Versamet Union y un microscopio electrónico de barrido (MEB) Philips XL30. El análisis puntual (microanálisis) fue realizado utilizando espectrometría de rayos-X por dispersión de energía (EDAX 4Dix) acoplado al MEB. Además, observaciones de MEB fueron utilizadas para evaluar los cambios en el estado superficial del mineral después de 4 y 10 semanas de alteración.

Para el análisis químico se realizó una digestión de las muestras de pirita, utilizando agua regia. El análisis de los metales en solución se realizó por Absorción Atómica utilizando un espectrómetro Perkin-Elmer 5000, el azufre elemental fue determinado en un analizador carbón-azufre marca Leco 244 SC, y el análisis del azufre total fue realizado por espectroscopia de plasma inducido (ICP) utilizando un espectrómetro Jarell Ash Iris 25. El contenido de sulfatos fue determinado por el método gravimétrico estándar. Finalmente, el contenido de sulfuro fue evaluado por diferencia entre el contenido de sulfato y azufre total en la muestra.

\section{Estudio electroquímico}

Se utilizó una celda típica de tres electrodos a temperatura ambiente y atmósfera inerte. El electrodo de trabajo fue un electrodo de pasta de carbón modificado con el mineral en estudio al 50\% en peso (CPE-Mineral), una barra de grafito fue utilizada como contra-electrodo y como electrodo de referencia se utilizó un electrodo saturado de sulfatos, al cual están referidos todos los potenciales reportados en este trabajo. El electrolito soporte fue una solución de $0.1 \mathrm{M} \mathrm{NaNO}_{3} \mathrm{pH}=6.5$. Un potenciostato PAR 273 conectado a una PC fue utilizado para obtener las respuestas voltamperométricas.

\section{Alteración del mineral}

Con el fin de caracterizar la evolución de la reactividad del mineral bajo distintas condiciones superficiales, se realizaron estudios electroquímicos y mineralógicos a muestras de mineral "fresco" y a diferentes estados superficiales generados a través de periodos de alteración. El proceso de alteración fue cíclico, durante el cual las muestras fueron sometidas a $3 \mathrm{~h}$ de inundación con una solución que simula el agua de lluvia (agua desionizada, $\mathrm{pH}=$ 5.5 ajustado con $\mathrm{CO}_{2}$ ), y a 3 días de exposición a condiciones ambientales. Se obtuvieron muestras de mineral a varios tiempos de alteración y muestras de solución en cada lixiviación (inundación) a las cuales se les analizó el pH y contenido de metales

\section{RESULTADOS Y DISCUSIÓN}

\section{Análisis de la reactividad de piritas ${ }^{13}$}

\section{Caracterización mineralógica}

La composición mineralógica de cada muestra se presenta en la Tabla 1. Estos resultados indican que las muestras de pirita Huckleberry y Louvicourt 1 presentaron un contenido limitado de impurezas sulfurosas, las cuales consistieron en pequeñas inclusiones de esfalerita $(\mathrm{ZnS})$, galena $(\mathrm{PbS})$, calcopirita $\left(\mathrm{CuFeS}_{2}\right)$ y arsenopirita (FeAsS) (Tabla 1). Las muestras de pirita de Louvicourt 2 y Tizapa presentaron impurezas de esfalerita, calcopirita, galena, así como arsenopirita, las cuales ocurren principalmente como partículas externas llenando los espacios entre partículas de pirita (Figura 1). Algunas de estas impurezas también se presentaron como inclusiones dentro de los cristales de pirita. Las muestras de pirita de Brunswick y Zimapán presentaron la más alta cantidad de impurezas de esfalerita, galena y arsenopirita, las cuales ocurren principalmente como inclusiones dentro de la pirita. Sin embargo, debido al tamaño de partícula de la muestra de Brunswick, algunas inclusiones o intercrecimientos de sulfuros en la pirita estaban expuestos.

La reactividad, o capacidad de oxidarse de la pirita se estudia normalmente en función del contenido y tipo de impurezas en su red cristalina. Sin embargo, en este trabajo no fue posible realizar tal comparación, debido a la imposibilidad de remover las impurezas existentes entre las partículas de pirita o incluidas en ellas. Por tal razón, en este estudio se evalúa el efecto global de las impurezas sobre la reactividad de la pirita y en su cinética de alteración.

\section{Estudio electroquímico}

En este trabajo, la variación de la reactividad de piritas se basa en la comparación de sus comportamientos voltamperométricos, por lo cual fue muy importante la reproducibilidad de la respuesta voltamperométrica de cada muestra, lográndose ello al iniciar cada experimento a partir del potencial de corriente nula (PCN). Un promedio de los valores de PCN para cada una de las muestras se presenta en la tabla 2. Estos valores de PCN parecen seguir una tendencia similar a la relación de impurezas en las muestras de pirita. Una relación similar entre el PCN y el contenido de impurezas ha sido reportada por Doyle y Mirza ${ }^{14}$. De tal manera, las piritas Huckleberry y Louvicourt 1 presentan valores similares de PCN. Por otra parte, Tizapa y Louvicourt 2, también presentan similar

Tabla 1. Composición química de las muestras piritosas (en \% en peso)

\begin{tabular}{|c|c|c|c|c|c|c|}
\hline & Huckleberry & Louvicourt 1 & Louvicourt 2 & Tizapa & Zimapán & Brunswick \\
\hline $\mathrm{Pi}$ & 96.9 & 84.5 & 80.4 & 95.5 & 89.1 & 80.4 \\
\hline Cpi & 0.1 & 0.1 & $<0.1$ & 2.3 & $<0.1$ & 0.92 \\
\hline $\mathrm{Ga}$ & $<0.1$ & $<0.1$ & 0.1 & 0.1 & 1.6 & 9.2 \\
\hline Esf & $<0.1$ & $<0.1$ & 1.7 & 0.9 & 1.2 & 4.3 \\
\hline Api & $<0.1$ & 0.1 & 0.1 & 0.4 & 2.6 & 0.6 \\
\hline MNS & 3.0 & 15.0 & 17.7 & 0.8 & 5.5 & 4.5 \\
\hline
\end{tabular}

$\mathrm{Pi}=$ Pirita Cpi $=$ Calcopirita $; \mathrm{Ga}=$ Galena Esf = Esfalerita; Api = Arsenopirita; MNS Minerales no sulforosos 
Tabla 2. Parámetros electroquímicos asociados a la respuesta electroquímica del CPE-Mineral para muestras de pirita sin alterar y después de 4 y 10 semanas de alteración; la caracterización electroquímica fue realizada en $0.1 \mathrm{M} \mathrm{NaNO}_{3}$

\begin{tabular}{|c|c|c|c|c|c|c|c|c|c|c|}
\hline \multirow[t]{2}{*}{ Muestra } & \multirow{2}{*}{$\begin{array}{c}\mathrm{PCN} \\
(\mathrm{V})\end{array}$} & \multicolumn{3}{|c|}{$\mathrm{E}_{\mathrm{I}=10 \mu \mathrm{A}}(\mathrm{V} / \mathrm{SSE})$} & \multicolumn{3}{|c|}{ Relación I/E $(\mu \mathrm{A} / \mathrm{mV})$} & \multicolumn{3}{|c|}{$\mathrm{Q}(\mathrm{mC})$} \\
\hline & & Sin Lixiviar & 4 sem. & 10 sem. & Sin lixivuar & 4 sem. & 10 sem. & Sin lixiviar & 4 sem. & 10 sem. \\
\hline Huckleberry & -0.27 & 0.37 & 0.43 & 0.43 & 1.86 & 1.38 & 1.37 & 5.84 & 3.27 & 3.16 \\
\hline Louvicourt 1 & -0.27 & 0.40 & 0.46 & 0.49 & 1.54 & 1.20 & 0.71 & 4.52 & 2.79 & 1.57 \\
\hline Louvicourt 2 & -0.19 & 0.49 & 0.43 & 0.46 & 0.70 & 1.24 & 0.94 & 1.50 & 3.15 & 2.17 \\
\hline Tizapa & -0.18 & 0.47 & 0.42 & 0.49 & 0.98 & 1.38 & 0.64 & 2.13 & 2.50 & 1.49 \\
\hline Zimapán & -0.17 & 0.44 & 0.46 & 0.47 & 1.47 & 1.22 & 1.25 & 3.47 & 2.79 & 2.18 \\
\hline Brunswick & -0.29 & 0.44 & 0.43 & 0.41 & 0.44 & 1.33 & 1.62 & 1.41 & 3.32 & 4.78 \\
\hline
\end{tabular}

$\mathrm{PCN}=$ potencial de corriente nula del CPE-Mineral; Relación I/E = corriente obtenida en función del incremento de potencial; $\mathrm{E}_{\mathrm{I}=10 \mu \mathrm{A}}=$ potencial requrido para alcanzar $10 \mu \mathrm{A}$ durante el barrido directo en el processo de oxidación de pirita; $\mathrm{Q}=$ carga asociada, evaluada a partir del área bajo la curva del processo de oxidación de pirita

PCN, aunque el contenido de MNS difiere enormemente, lo cual es ciertamente debido a que estas especies no son electroactivas. Finalmente, Zimapán y Brunswick presentan un PCN diferente a pesar del mismo contenido de pirita, lo cual podría deberse al efecto de los distintos tipos de ocurrencia de las impurezas (inclusiones o llenando intersticios) y/o a la cantidad de cada una de ellas, como será descrito posteriormente.

En la Figura 2 se presenta la comparación de los voltamperogramas obtenidos con cada una de las muestras de pirita sin alteración, iniciando el barrido de potencial en dirección positiva. En la Figura 2a se observa que el comportamiento voltamperométrico es similar para todas las muestras, sin embargo el potencial donde inicia el proceso de oxidación de la pirita es diferente
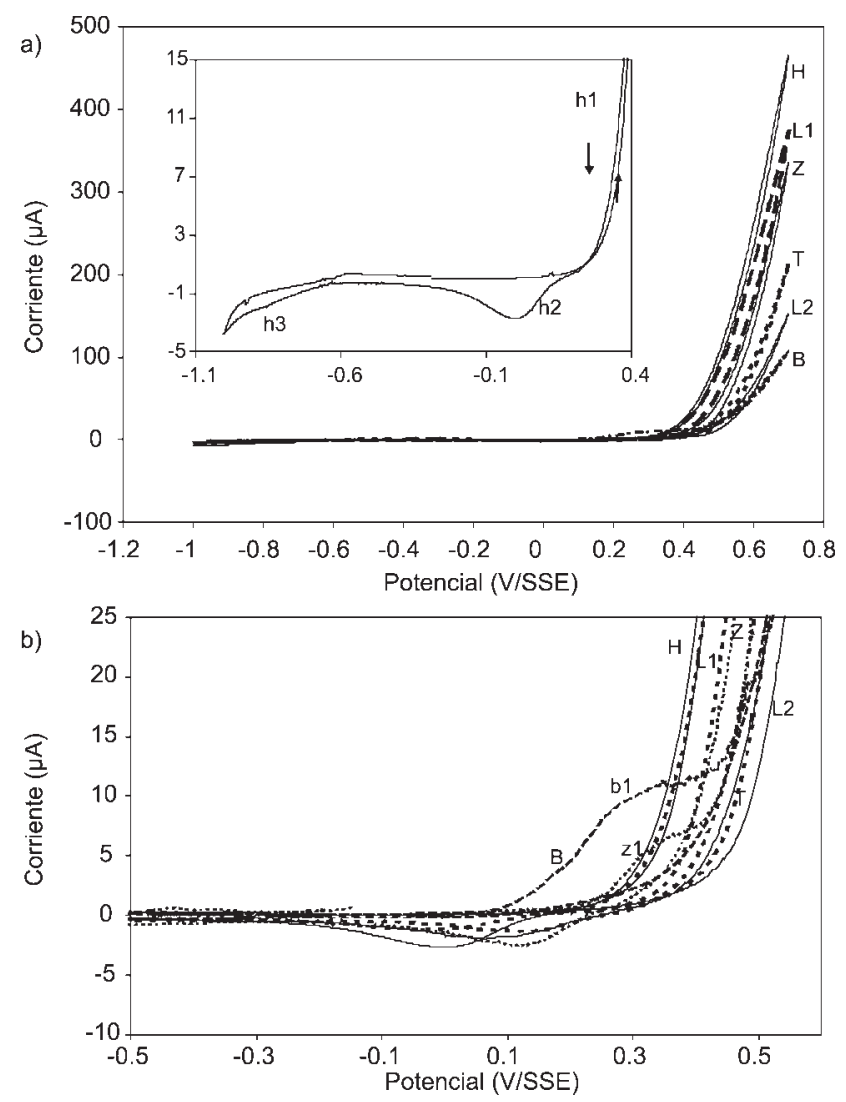

Figura 2. Voltamperogramas típicos obtenidos para las muestras de pirita de distinta procedencia: H, Huckleberry; L1, Louvicourt 1; L2 Louvicourt 2; T, Tizapa; Z, Zimapán; B, Brunswick. Los voltamperogramas fueron obtenidos a partir de un CPE-pirita al 50\% en 0.1M NaNO a $20 \mathrm{mV} \mathrm{s}^{-1}$. (a) Respuesta voltamperométrica completa, (b) zona de baja corriente de los voltamperogramas para cada muestra. En la Figura 2b se presenta una vista de la zona de baja corriente de los voltamperogramas de la Figura 2a. En esta figura se observa que el comportamiento voltamperométrico de la pirita Zimapán (curva Z) y la muestra de Brunswick (curva B) presenta un pico en $\sim 0.3 \mathrm{~V}$, anterior al proceso de oxidación de la pirita en $0.44 \mathrm{~V}$.

De acuerdo a la caracterización mineralógica, la pirita Huckleberry es la pirita más pura (97\% de pirita), por lo cual el voltamperograma correspondiente a esta muestra (recuadro de la Figura 2a) será considerado como el comportamiento típico de la pirita en $0.1 \mathrm{M} \mathrm{NaNO}_{3}$. En este voltamperograma se observa un pico alto de oxidación que inicia en $0.3 \mathrm{~V}$. Durante el barrido inverso se detectan dos picos de reducción, uno entre 0.14 y $-0.15 \mathrm{~V}$ y otro a $-0.7 \mathrm{~V}$ (recuadro). Aunque el estudio de los procesos catódicos podría aportar información sobre la naturaleza de los productos de oxidación de la pirita, este trabajo está enfocado solo al estudio del comportamiento anódico, debido a su obvia relación con la reactividad de la pirita.

En la mayoría de los estudios electroquímicos, las respuestas voltamperométricas son usualmente descritas en función de un potencial de pico. Debido a que en la respuesta voltamperométrica obtenida para la pirita no se presenta un pico como tal, en este trabajo se emplean como base de comparación los siguientes parámetros: (i) el potencial requerido para alcanzar, durante el barrido directo, una corriente de $10 \mu \mathrm{A}\left(\mathrm{E}_{\mathrm{I}=10 \mu \mathrm{A}}\right)$; (ii) la capacidad de oxidación de la pirita relativa al potencial impuesto, dada por la relación I/E, calculada a partir de la pendiente de la respuesta corriente-potencial durante el barrido directo, en un intervalo de potencial $0.55-0.66 \mathrm{~V}$; (iii) la carga asociada al proceso de oxidación $(\mathrm{Q})$, obtenida a partir del área bajo la curva del proceso de oxidación. En la tabla 2 se presenta los valores de estos parámetros, los cuales facilitan la comparación cuantitativa de la reactividad de las muestras. Estos parámetros electroquímicos $\left(\mathrm{E}_{\mathrm{I}}\right.$, I/E y Q) indican que la pirita Huckleberry requiere el menor potencial para que se presente el proceso de oxidación; además, presenta los valores más altos de carga asociada (Q) y de capacidad relativa de oxidación (relación I/E). De acuerdo a estos resultados (Tabla 2) se puede proponer que la pirita Huckleberry es la pirita más reactiva de todas las analizadas y que el grado de reactividad de las muestras de pirita disminuye en el siguiente orden: Huckleberry, Louvicourt 1, Zimapán, Tizapa, Louvicourt 2 y Brunswick. Por tal razón, siendo la pirita Brunswick la menos reactiva es la que posee una relación I/E menor. Es importante decir que los valores de $E_{I}$ no presentan una misma secuencia que los valores de $\mathrm{I} / \mathrm{E}$ o los de Q, lo cual puede ser atribuido al comportamiento electroquímico de las impurezas presentes en las muestras, tal como lo señalan los picos z1 y b1 (Figura 2b). 

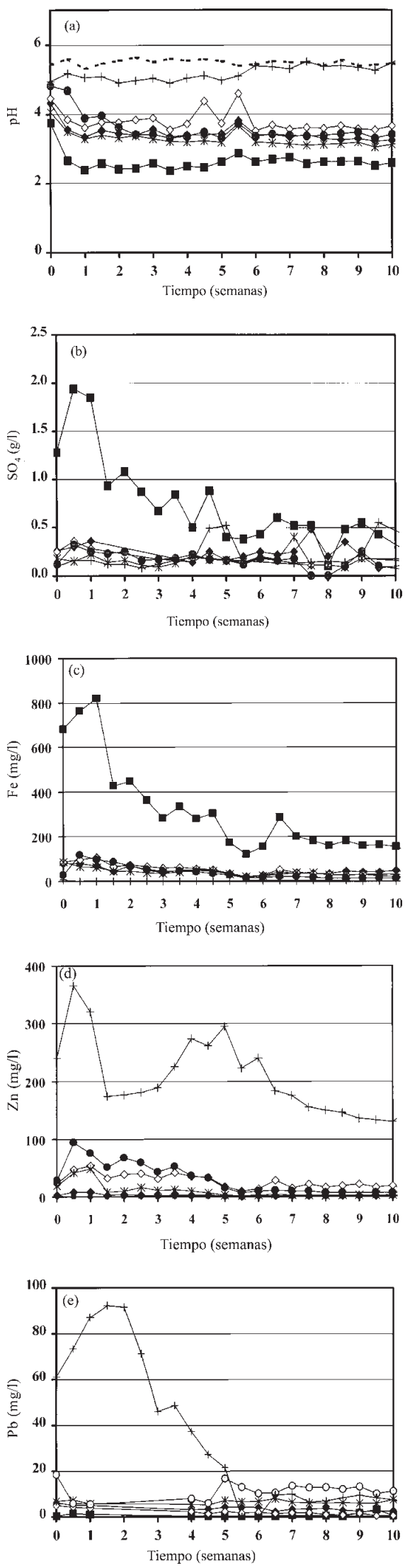

Figura 3. Evolución del $p H(a)$, sulfatos (b), $\mathrm{Fe}(c), \mathrm{Zn}(d)$ y $\mathrm{Pb}(\mathrm{e})$ disueltos en el lixiviado, en función del tiempo de alteración de las muestras de: (口) Huckleberry, ( ) Louvicourt 1, ( ) Louvicourt 2, (*) Tizapa, (৩) Zimapán y (+) Brunswick. La línea punteada en (a) corresponde al pH de la solución inicial de lixiviación
Alteración de piritas y evolución de la reactividad

Los análisis químicos del lixiviado ( $\mathrm{Fe}, \mathrm{Zn}, \mathrm{Pb}$, y sulfatos) y la evolución de su $\mathrm{pH}$, presentaron distintas tendencias para cada muestra, lo cual sugiere cinéticas de disolución diferente para cada pirita (Figura 3). El pH se estabiliza después de una rápida disminución durante la primera semana de alteración, excepto para Brunswick (Figura 3a). El lixiviado de esta muestra, la cual es la menos reactiva de acuerdo al estudio voltamperométrico, presenta un pH similar al de la solución empleada para la inundación (Figura 3a). Por otra parte, el lixiviado de la muestra más reactiva (Huckleberry), presenta el pH más bajo (Figura 3a). De igual forma la concentración más alta de sulfato y hierro en solución es mayor para la pirita Huckleberry (Figura 3).

La disolución de cinc es mayor para las muestras con mayor contenido de esfalerita disponible en la muestra (Figura 3d). La concentración de cinc en los lixiviados de Tizapa y Zimapán presenta una caída después de 5 semanas de alteración, mientras que para los lixiviados de Brunswick y Louvicourt 2 disminuye lentamente. El plomo, generado por la disolución de galena en las muestras, presenta su más alta disolución durante las primeras 5 semanas para la muestra Brunswick (Figura 3e). Posteriormente, se estabiliza en valores similares a los obtenidos para las muestras de Zimapán y Tizapa $\left(\sim 10 \mathrm{mg} \mathrm{L}^{-1}\right)$.

La evolución de la reactividad electroquímica de las muestras sin alteración y después de 4 y 10 semanas de alteración, es estudiada analizando la parte anódica (de -0.1 a 0.7 V) del voltamperograma completo y los parámetros electroquímicos respectivos $\left(\mathrm{E}_{\mathrm{I}}, \mathrm{I} / \mathrm{E}\right.$ y $\left.\mathrm{Q}\right)$. La figura 4 presenta la evolución de la reactividad de la pirita de Huckleberry, por medio de los cambios en el comportamiento voltamperométrico, en función del tiempo de alteración. La curva $a$ corresponde al mineral sin alterar, y las curvas $b$ y $c$ representan el comportamiento voltamperométrico de la muestra después de 4 y 10 semanas de alteración, respectivamente. Después de 4 semanas de alteración, se presenta una disminución en la relación I/E (capacidad de oxidación) y una variación de $+60 \mathrm{mV}$ en el valor de $\mathrm{E}_{\mathrm{l}}$, con respecto a la pirita sin alterar (Tabla 2, Figura 4). Esta misma tendencia se mantiene hasta la décima semana de alteración (curva $c$ ). Los cambios observados en el comportamiento voltamperométrico de la pirita antes y después de la alteración pueden ser explicados de la siguiente manera: durante los ciclos de alteración, se forman precipitados de especies de hierro los cuales constituyen una capa pasivante que retarda y disminuye el proceso de oxidación, es decir, un sobrepotencial mayor es necesario para que el proceso de oxidación inicie. La formación de este tipo de precipitados sobre pirita ha sido reportada anteriormente por otros autores ${ }^{15,16}$. La pirita Louvicourt 1 presentó un

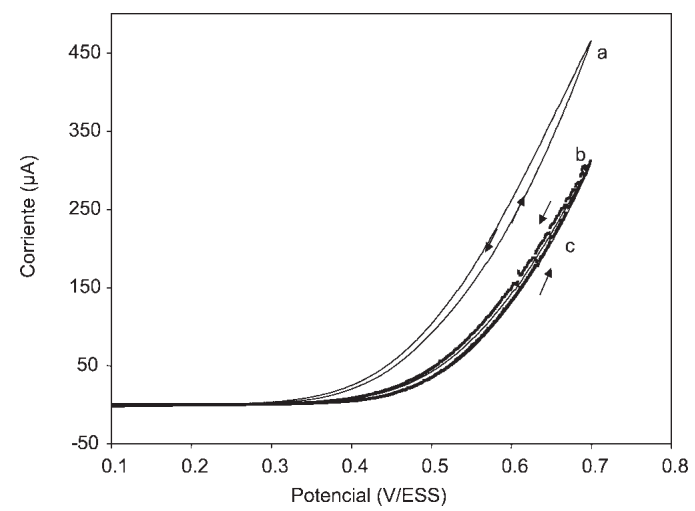

Figura 4. Evolución del comportamiento voltamperométrico de la pirita Huckleberry a diferentes etapas de alteración: (a) sin alterar, (b) 4 semanas $y$ (c) 10 semanas. CPE-Mineral al $50 \%$ en $0.1 \mathrm{M} \mathrm{NaNO}{ }_{3}$. El barrido de potencial fue iniciado en dirección positiva a $20 \mathrm{mV} \mathrm{s}^{-1}$ 
comportamiento similar a la pirita Huckleberry, como se puede observar a partir de los parámetros electroquímicos de la Tabla 2.

La muestra de Louvicourt 2 (Figura 5) presenta una evolución del comportamiento voltamperométrico distinta a la de Huckleberry, ya que después de 4 semanas de alteración (curva $b$ ) se presenta una ganancia en la reactividad electroquímica de esta muestra, con un desplazamiento negativo de $\sim 70 \mathrm{mV}$ en el valor de $\mathrm{E}_{\mathrm{I}} \mathrm{y}$ un incremento de 0.7 a $1.24 \mu \mathrm{A} \mathrm{mV}^{-1}$ en el $\mathrm{I} / \mathrm{E}$, en comparación a la pirita sin alterar. La respuesta obtenida para después de 10 semanas de alteración (curva $c$ ), presenta una pasivación de la pirita con un desplazamiento positivo de $\sim 40 \mathrm{mV}$ con respecto a la pirita con 4 semanas de alteración. Una ganancia neta en la reactividad, reflejada por un desplazamiento negativo total de $\sim 30 \mathrm{mV}$ con respecto a la pirita sin alterar, resultó después de 10 semanas. Esta reactivación esta ciertamente asociada a la perdida de una protección galvánica de las impurezas sobre la pirita, lo cual fue demostrado por una alta disolución de cinc y una baja disolución de hierro durante las primeras semanas, es decir, la pirita se disuelve más lentamente que las impurezas. Cuando las impurezas son removidas por el proceso de alteración durante las primeras 4 semanas se pierde la protección galvánica y se favorece la reactivación de la pirita. Las observaciones de MEB para muestras con 4 semanas de alteración, mostraron partículas de esfalerita altamente corroídas y partículas de pirita con superficies no corroídas y relativamente libres de precipitados de hierro (Figura 6). Para 10 semanas de alteración, la pirita presenta una disminución en su reactividad con tendencia similar a la pirita Huckleberry, y la aparición de patrones de corrosión en su superficie (Figura 6). El incremento de la oxidación de la pirita implica un incremento de iones de hierro y azufre en la solución, lo cual promueve la formación de precipitados de hierro y/o de azufre sobre la superficie del mineral. La pirita Tizapa presentó un comportamiento similar al descrito para Louvicourt 2, pero la ganancia de reactividad fue en menor grado y la pasivación a las 10 semanas de alteración fue mayor (Tabla 2).

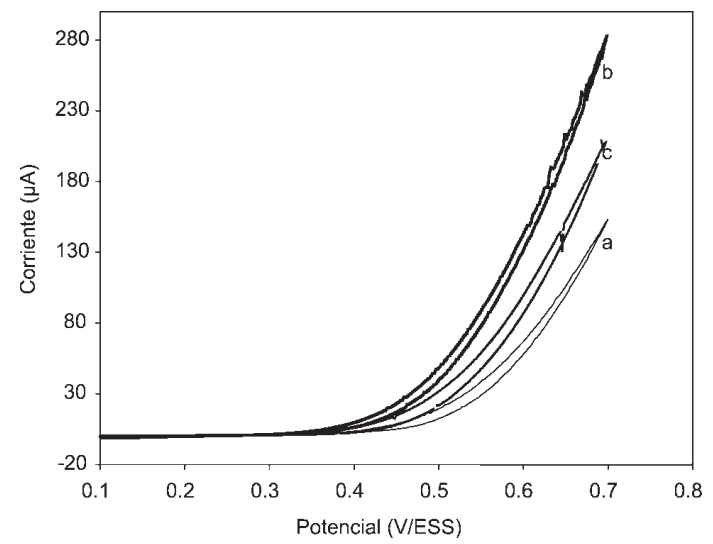

Figura 5. Evolución del comportamiento voltamperométrico de la pirita Louvicourt 2 a diferentes etapas de alteración: (a) sin alterar, (b) 4 semanas y (c) 10 semanas de alteración. CPE-Mineral al $50 \%$ en $0.1 \mathrm{M} \mathrm{NaNO}$. El barrido de potencial fue iniciado en dirección positiva a $20 \mathrm{mV} \mathrm{s}^{-1}$

La pirita Zimapán, al igual que la pirita Huckleberry, presenta una pasivación de la superficie (Figura 7), tal como lo indican los cambios en los valores de $\mathrm{I} / \mathrm{E}_{\mathrm{y}} \mathrm{E}_{\mathrm{I}}$ (Tabla 2). Una pequeña variación de estos parámetros se observa entre 4 y 10 semanas, con un desplazamiento positivo total de $\sim 30 \mathrm{mV}$, con respecto a la pirita $\sin$ alterar. A diferencia de la pirita Huckleberry, la pirita Zimapán presenta un incremento en el ancho del proceso principal de oxidación conforme aumenta el tiempo de alteración. Esto se debe a la acción conjunta de la formación de picaduras y a la formación de una capa delgada de precipitados sobre la superficie del mineral, tal como se
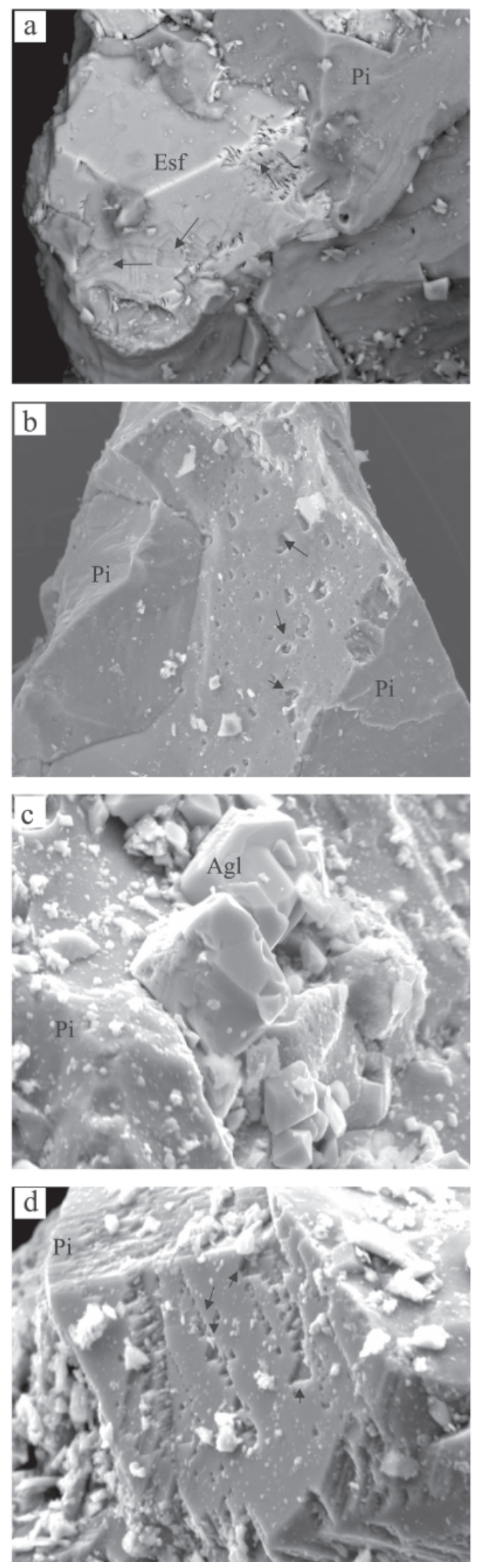

Figura 6. Imágenes por MEB para diferentes muestras de piritas: (a) esfalerita corroída y superficie lisa de pirita en la muestra Louvicourt 2, a 10 semanas de lixiviación, (b) picaduras de corrosión en la superficie de pirita Louvicourt 2 a 10 semanas de lixiviación, (c) galena completamente remplazada por anglesita en la muestra de Zimapán a 10 semanas de lixiviación, y (d) picaduras de oxidación en la superficie de la pirita de Zimapán a 4 semanas de lixiviación. $\mathrm{Pi}=$ Pirita, Esf = Esfalerita y Agl = anglesita 


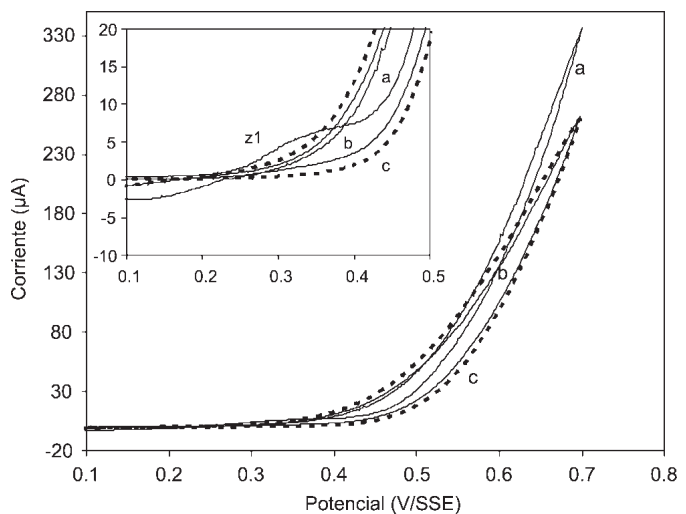

Figura 7. Evolución del comportamiento voltamperométrico de la pirita Zimapán a diferentes etapas de alteración: (a) sin alterar, (b) 4 semanas y (c) 10 semanas. CPE-Mineral al $50 \%$ en $0.1 \mathrm{M} \mathrm{NaNO}_{3}$. El barrido de potencial fue iniciado en dirección positiva a $20 \mathrm{mV} \mathrm{s}^{-1}$

observó en las imágenes por MEB (Figura 6). Una vez que la capa delgada de pasivación se rompe por la acción del potencial impuesto, una mayor área electroactiva está disponible, por efecto de las picaduras en la superficie de la pirita. A pesar de que la muestra Zimapán contiene una cantidad considerable de esfalerita, esta muestra no sigue el comportamiento observado para las muestras Louvicourt 2 y Tizapa, lo cual probablemente se debe que la esfalerita en la muestra Zimapán ocurre como inclusiones dentro de las partículas de pirita (Figura 2) y no entra en contacto con la solución, lo que evita la formación del par galvánico con la pirita. Es importante notar la desaparición del pico z1 con el avance del proceso de alteración (recuadro de la Figura 7), lo cual es provocado por la disolución de la galena, uno de los minerales con mayor velocidad de oxidación ${ }^{17}$, siendo completamente reemplazada por anglesita, $\mathrm{PbSO}_{4}$ (Figura 6).

El concentrado piritoso de Brunswick presentó una ganancia en su reactividad, como lo demuestra el desplazamiento negativo de $\sim 30 \mathrm{mV}$ del $\mathrm{E}_{\mathrm{I}}$ entre la muestra sin lixiviar y la muestra con 10 semanas de alteración. De igual manera, los parámetros Q e I/E, indican el incremento en la reactividad del mineral, en función del tiempo de alteración (Tabla 2). Sin embargo, su lixiviado presenta una muy baja concentración de hierro aun después de 10 semanas de alteración, lo cual es consecuencia de una baja reactividad de la pirita en la muestra. Como ha sido mostrado en este trabajo, la protección galvánica que ofrece la esfalerita depende de su accesibilidad. Las partículas liberadas de esfalerita presentes en el concentrado de Brunswick (Figura 1c), no permiten la oxidación de la pirita y la consecuente generación de precipitados de hierro que pasivan la superficie. Por tal razón, la respuesta voltamperométrica de la muestra de Brunswick presenta una ganancia progresiva considerable en la reactividad de la pirita (Figura 8, Tabla 2).

La metodología aquí descrita fue utilizada para estudiar muestras de pirita con diversas composiciones y tipos de impureza, lográndose resultados reproducibles, con respecto a la evolución de la reactividad de la pirita, para aquellas muestras con tipo y cantidad de impurezas similares a las descritas en este trabajo. Con estos resultados fue posible deducir, que la presencia de sulfuros minerales $(\mathrm{PbS}, \mathrm{ZnS}$, etc.) en contacto con la pirita es el principal factor que afecta la reactividad de la pirita durante el inicio de su oxidación. Para instancias más avanzadas, el recubrimiento de la pirita por una capa pasivante vendría a ser el parámetro más importante que define la reactividad de la pirita y por lo tanto la disponibilidad de los agentes oxidantes. Más detalles se encuentran descritos en la referencia 13.

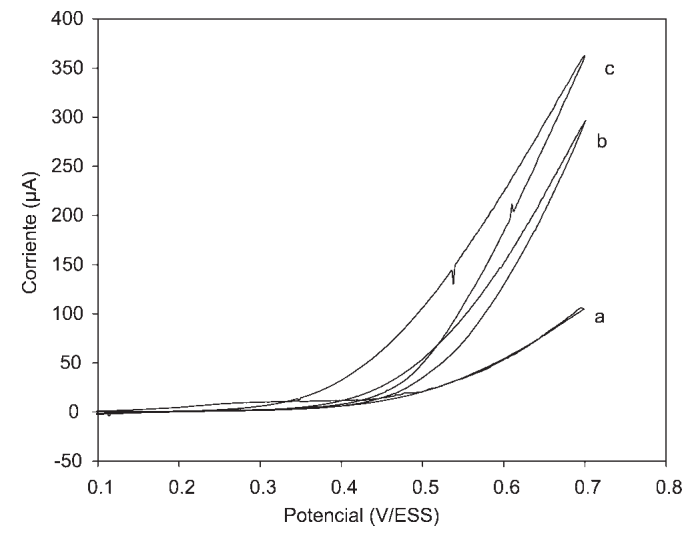

Figura 8. Evolución del comportamiento voltamperométrico de la muestra de Brunswick a diferentes etapas de alteración: (a) sin alterar, (b) 4 semanas $y$ (c) 10 semanas. CPE-Mineral al 50\%. El barrido de potencial fué iniciado en dirección positiva a $20 \mathrm{mV} \mathrm{s}^{-1}$

\section{Análisis de la reactividad de pirrotita ${ }^{18}$}

Debido a la baja disponibilidad de fases puras de pirrotita, en este estudio sólo se analizó una muestra. Sin embargo, es importante decir que un estudio comparativo de distintas muestras de pirrotita aportaría mayor información sobre los factores que afectan su reactividad. En este trabajo se estudió el efecto de los cambios superficiales en la reactividad de la pirrotita.

\section{Caracterización mineralógica}

La reconstrucción mineralógica de la muestra determinó que ésta contenía $97.9 \%$ pirrotita $\left(\mathrm{Fe}_{1-\mathrm{x}} \mathrm{S}\right), 0.9 \% \mathrm{ZnS}, 0.7 \% \mathrm{PbS}, 0.1 \%$ FeAsS y $0.4 \%$ de minerales no sulfurosos. Las impurezas ocurren como inclusiones en el cristal de pirrotita. La relación media atómica de la pirrotita es $\mathrm{S}: \mathrm{Fe} 1.12\left(\mathrm{Fe}_{0.89} \mathrm{~S}\right)$.

\section{Estudio electroquímico}

En la Figura 9 se presenta el comportamiento voltamperométrico de la pirrotita sin alterar, el cual será empleado como patrón de comparación en el análisis de las muestras a distintos tiempos de alteración. Cuando el barrido es iniciado en dirección positiva se observa un pico de oxidación (O1) el cual inicia en $\sim 0.3 \mathrm{~V}$ (Figura 9a). Dicho pico presenta un proceso de pasivación, seguida por un proceso de oxidación transpasiva (OT). Cuando el potencial es invertido, se observan los picos de reducción R1 ($0.2 \mathrm{~V}), \mathrm{R} 2(-1.05 \mathrm{~V})$ y R3 (-1.3V). Además en la parte final del ciclo se observa un segundo pico de oxidación $(\mathrm{O} 2)$ en $-0.47 \mathrm{~V}$ (Figura 9).

De acuerdo a un estudio previo sobre el mecanismo de oxidación de la pirrotita, empleando voltamperometría cíclica a distintas velocidades de barrido y variando el potencial de inversión ${ }^{18}$, y apoyándose en datos reportados por otros autores ${ }^{5,8}$, se pudo establecer que el mecanismo de oxidación electroquímica de pirrotita (pico O1) involucra las siguientes etapas.

Etapa 1: $\mathrm{Fe}_{0.89} \mathrm{~S} \rightarrow \mathrm{Fe}_{0.89-\mathrm{x}} \mathrm{S}+\mathrm{xFe}^{2+}+2 \mathrm{xe}^{-}$

Etapa 2: $\quad \mathrm{Fe}_{0.89-\mathrm{x}} \mathrm{S} \rightarrow(1-\mathrm{x}) \mathrm{Fe}^{2+}+1.12 \mathrm{~S}^{\circ}+2(1-\mathrm{x}) \mathrm{e}^{-}$

Etapa 3: $\quad \mathrm{Fe}^{2+}+3 \mathrm{H}_{2} \mathrm{O} \rightarrow \mathrm{Fe}(\mathrm{OH})_{3}+3 \mathrm{H}^{+}+\mathrm{e}^{-}$

Según el estudio realizado variando el potencial de inversión positivo, la reducción de especies de hierro se da en R1, mientras 


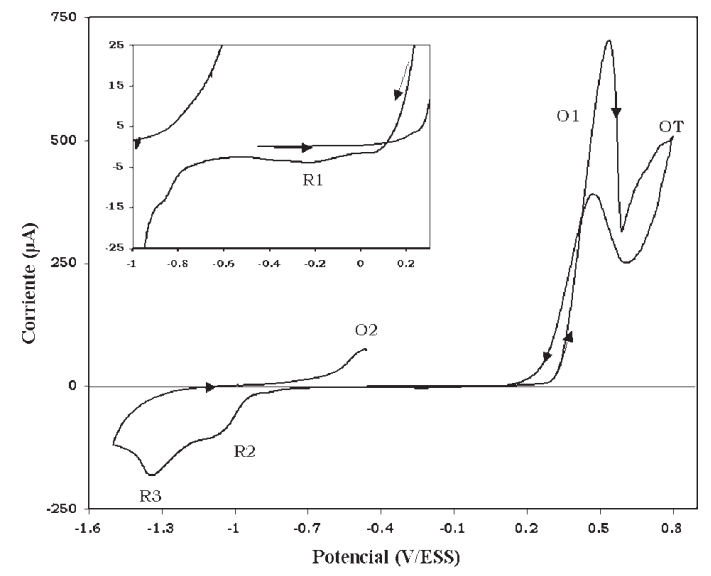

Figura 9. Voltamperograma típico obtenido para pirrotita a partir de un CPEpirrrotia $50 \%$ en $0.1 \mathrm{M} \mathrm{NaNO}$. El barrido de potencial fue iniciado en dirección positiva a partir del potencial de corriente nula, $v=20 \mathrm{mV} \mathrm{s}^{-1}$

que el pico R2 esta asociado a la reducción de especies de azufre elemental y el pico R3 corresponde a al reducción del $\mathrm{Fe}_{0.89-\mathrm{x}} \mathrm{S}$.

Análisis electroquímico de la evolución del estado superficial de pirrotita

Considerando que la evolución del estado superficial de pirrotita durante el proceso de alteración, debe estar relacionada con la evolución de la reactividad del mineral, se realizó un estudio voltamperométrico a muestras con distintos tiempos de alteración. En la figura 10, se presenta la evolución del comportamiento voltamperométrico de pirrotita después de 2, 4, y 10 semanas de alteración, cuando el barrido es iniciado en dirección positiva. En esta figura se puede observar que el proceso de oxidación $\mathrm{O} 1$, y el potencial donde este inicia disminuyen con el tiempo de alteración (Figura 10). Por otra parte la corriente involucrada en los picos de reducción $\mathrm{R} 1, \mathrm{R} 2$ y $\mathrm{R} 3$ incrementa, formando estos dos últimos un solo pico. Puesto que la magnitud de corriente involucrada en los picos de reducción incrementa con el tiempo de alteración, se podría considerar que las especies formadas durante la oxidación electroquímica de pirrotita (reacciones 5,6,7), son similares a las formadas durante la alteración química, un análisis a detalle a sido reportado anteriormente ${ }^{18}$.

De acuerdo a estos resultados, es presumible que el mecanismo de alteración química de la pirrotita sigue un mecanismo similar al de su oxidación electroquímica. Sin embargo, hasta este punto, es difícil establecer con certeza la etapa del mecanismo que tiene mayor efecto en la disminución de la reactividad de la pirrotita, esto debido a que las variaciones del proceso de oxidación electroquímica de las muestras alteradas sólo muestran cambios globales (pico O1), mientras que los cambios específicos no son observados con claridad.

Efecto de las especies superficiales en la reactividad de pirrotita

La formación de especies oxidada de hierro, azufre elemental, y el sulfuro deficiente en hierro fue corroborada utilizando DRX y MEB (Figura 11). El efecto de dichas especies se analizó por comparación de voltamperogramas obtenidos para muestras con 10 semanas de alteración a las cuales se les removió con lixiviación selectiva los hidróxidos de hierro y azufre elemental. De esta comparación (Figura 12) se puede establecer que la remoción de azufre elemental genera una superficie con comportamiento similar al de la pirrotita sin alterar, sin embargo el proceso de oxidación no logra la pasivación debido a que se ha generado una alta área
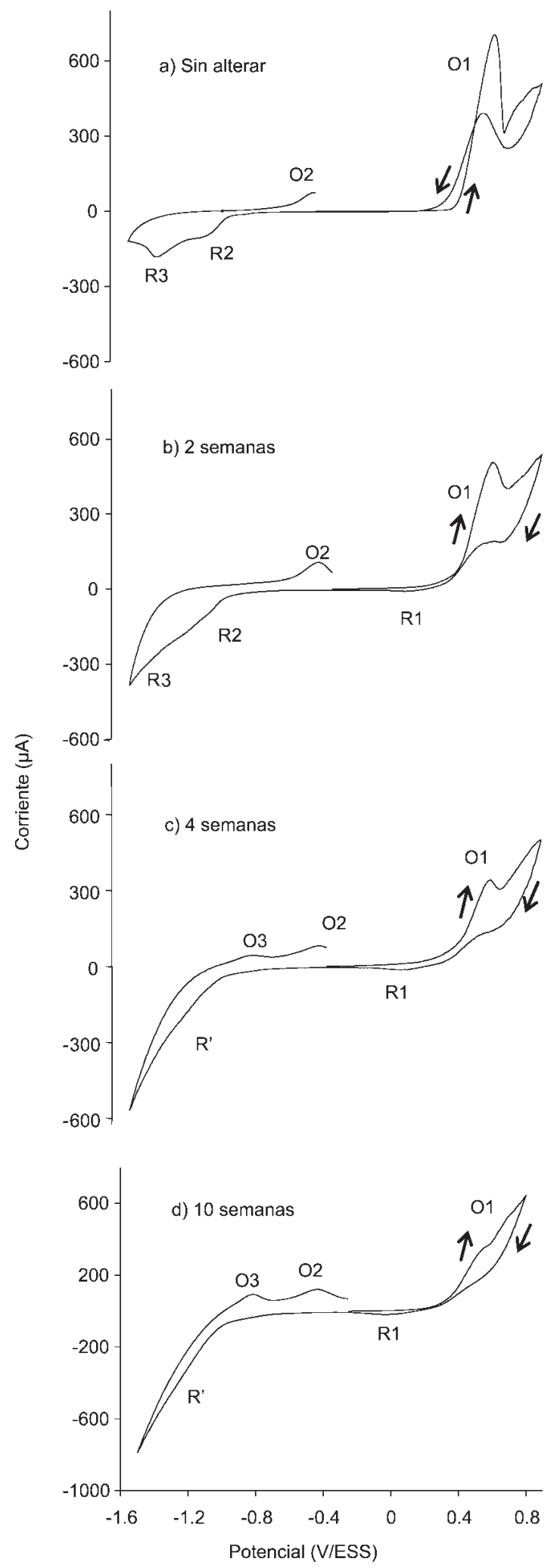

Figura 10. Evolución del comportamiento voltamperométrico de pirrotita a diferentes etapas de alteración: (a) sin alterar, (b) 2 semanas, (c) 4 semanas $y(d) 10$ semanas. CPE-Mineral al 50\%. El barrido de potencial fue iniciado en dirección positiva a $20 \mathrm{mV} \mathrm{s}^{-1}$

electroactiva durante la alteración. Por lo anterior se dedujo que la capa de azufre presenta un efecto mayor en la reducción de la reactividad de la pirrotita. 

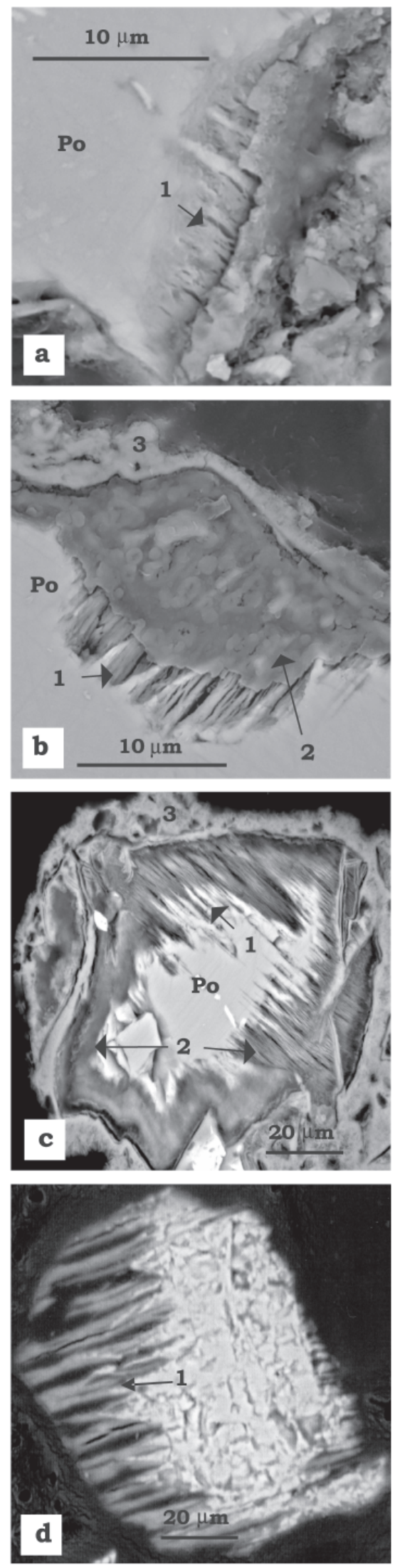

Figura 11. Imágenes de $M E B$ de partículas de pirrotita a diferentes etapas de alteración: (a) 2 , (b) 4 y (c) 10 semanas. Po = Pirrotita. Las zonas marcadas por los números son (1) estructura lamelar con contenido de hierro menor al de la pirrotita; (2) fase reniforme de azufre y (3) cristales constituidos por hierro y oxígeno

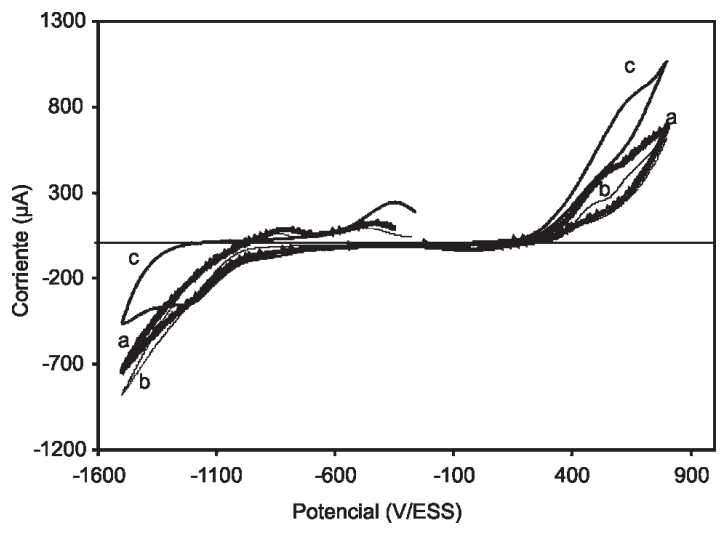

Figura 12. Respuesta voltamperométrica de la muestra de pirrotita después de 10 semanas de alteración cuando: (a) sin lavado, (b) las fases de $\mathrm{FeOOH}$ fueron eliminadas, (c) ambas fase $\mathrm{FeOOH}$ y $\mathrm{S}^{\circ}$ han sido eliminadas. El barrido de potencial fue iniciado en dirección positiva a $20 \mathrm{mV} \mathrm{s}$

\section{Análisis de la reactividad de residuos mineros ${ }^{19}$}

Con el fin de evaluar las perspectivas de aplicación de la metodología descrita para las muestras de pirita y pirrotita en los estudios de predicción de DAR, se analizó la evolución de un residuo minero sulfuroso. La composición de esta muestra fue $64.7 \%$ pirita, $2.1 \%$ esfalerita, $0.4 \%$ galena, $0.6 \%$ arsenopirita, $0.2 \%$ calcopirita, $0.1 \%$ tetraedrita, $0.5 \%$ calcita y el resto consistía en minerales no sulfuroso y cuarzo. Esta muestra resulto potencialmente generadora de DAR, de acuerdo a las pruebas estáticas ${ }^{19}$.

La comparación de los voltamperogramas obtenidos para esta muestra a diferentes tiempos de alteración se presenta en la Figura 13. En esta figura se observa que la muestra sin alterar presenta un comportamiento diferente al comportamiento típico de muestras que contienen pirita (curva a). Esto se debe a la generación de especies superficiales de ion ferroso durante el procesamiento industrial del mineral. La presencia de estas especies oxidadas provoca el comportamiento voltamperométrico singular observado en la Figura 13, el cual involucra tanto la oxidación de las especies oxidadas, como la oxidación de la pirita. Por lo tanto, los cambios observados en el comportamiento voltamperométrico después de 2 semanas (Figura 13), deben de estar asociados a la eliminación de estas especies electroactivas de la superficie del mineral. De acuerdo al análisis de las piritas y al contenido de impurezas de esta muestra de residuos mineros, se esperaría una protección galvánica para la pirita. Sin embargo, el análisis de la disolución de metales indicó que a pesar de una disolución preferencial de esfalerita en las primeras semanas de alteración, el contenido del hierro en solución se mantuvo constante durante todo el periodo de alteración, lo cual podría sugerir que la pirita se está disolviendo y que no se presenta la protección galvánica. Sin embargo, el análisis voltamperométrico reveló que si se presenta dicha protección y que permanece hasta antes de la semana 10 de alteración, después de lo cual se presenta la reactivación electroquímica de la muestra (Figura 13c). Es importante señalar que la evolución de la reactividad, observada por el análisis de la solución sería similar a los resultados que se obtendrían en las pruebas cinéticas de predicción de DAR, en las cuales se enmascararía la evolución real de la reactividad de la muestra. Es importante señalar que con el empleo de la voltamperometría, la evolución real de la reactividad de la muestra fue evaluada más adecuadamente. Más detalles sobre el estudio y aplicación de esta metodología a residuos mineros se encuentran en la referencia 19. 


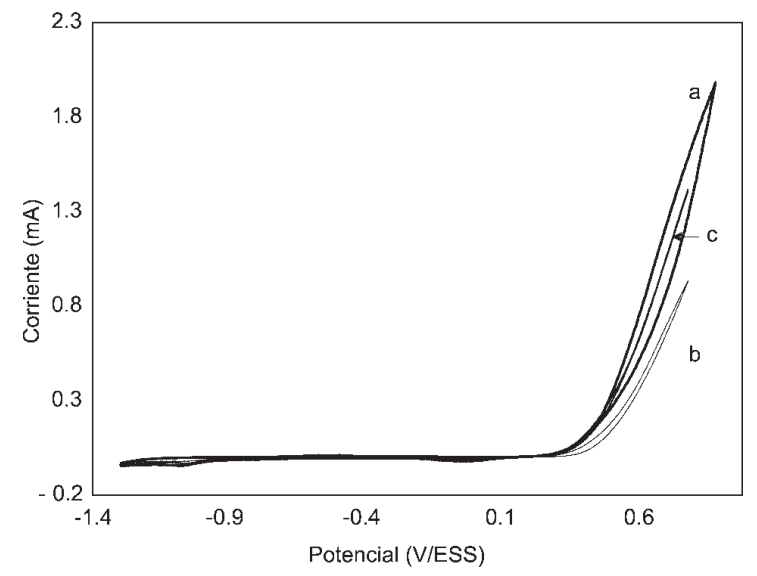

Figura 13. Evolución del comportamiento voltamperométrico de la muestra de residuos mineros a diferentes tiempos de alteración: (a) sin lixiviar, (b) 2 semanas y (c) 10 semanas. CPE-Mineral al $50 \%$ en $0.1 \mathrm{M} \mathrm{NaNO}_{3}$. El barrido de potencial fue iniciado en dirección positiva a $20 \mathrm{mV} \mathrm{s}^{-1}$

\section{CONCLUSIONES}

El empleo de voltamperometría cíclica de barrido triangular con electrodos de pasta de carbón, apoyada con las técnicas típicas de caracterización de minerales, ofrece una metodología efectiva para evaluar los factores que afectan la reactividad de los sulfuros de hierro a condiciones simuladas de intemperismo. Con la aplicación de esta metodología se pudo establecer que la presencia de impurezas y su interacción con la pirita son los factores que afectan principalmente la reactividad de este mineral. Para el caso de la pirrotita, se pudo establecer que la formación de capas de $\mathrm{FeOOH}$ y $\mathrm{S}^{0}$ en la superficie de la pirrotita, es el principal factor que afecta su reactividad. El establecer dichos factores no se hubiera podido lograr con el simple conocimiento de las características mineralógicas de las muestras y de los resultados de la aplicación de las pruebas cinéticas a las muestras, como se demostró en la aplicación de la metodología en el análisis de muestras de residuos mineros, en donde además se comprobó que los factores que afectan la reactividad de los sulfuros en los residuos mineros son los mismos que los determinados para muestras de sulfuros de hierro.

El conocimiento adquirido sobre el efecto específico de cada tipo de impureza en la capacidad oxidativa del mineral, así como de las capas superficiales desarrolladas sobre el mineral, puede ser utilizado como base para desarrollar un método de evaluación y predicción del potencial de generación de DAR de residuos mineros que contengan sulfuros minerales.

\section{REFERENCIAS}

1. Keith, C. N.; Vaughan, D. J. Em Environmental Mineralogy: Microbial Interactions, Anthropogenic Influences, Contaminated Land and Waste Management; Cotter-Howells, J. D.; Campbell, L. S.; Valsami-Jones, E.; Batchelder, M., eds.; Mineralogical Society of Great Britain \& Ireland; London, 2000, p. 117.

2. Nordstrom, D. K.; Alpers, C. N. Em The Environmental Geochemistry of Mineral Deposits. Part A: Processes, Techniques and Health Issues; Plumlee, G. S.; Logsdon, M. J., eds.; Society of Economic Geologists, Inc.; Littleton, USA, 1999, p. 133.

3. Schmiermund, R. L.; Drozd, M. A. Em Mining Environmental Handbook, Effects of Mining on the Environment and American Environmental Control on Mining; Marcus, J. J., ed.; Imperial College Press: London 1997, p. 599.

4. Janzen, M. P.; Nicholson, R. V.; Scharer, J. M.; Geochim. Cosmochim. Acta 2000, 64, 1511.

5. Hamilton, I. C.; Woods, R.; J. Electroanal. Chem. 1981, 118, 327.

6. Thomas, J. E.; Skinner, W. M.; Smart, R.; Geochim. Cosmochim. Acta 2001, $65,1$.

7. Buckley, A. N.; Woods, R.; Appl. Surf. Sci. 1985, $20,472$.

8. Mikhlin, Y. L.; Kuklinsky, A.V.; Pavlenko, N. I.; Geochim. Cosmochim. Acta 2002, 66, 4057

9. Morin, K. A.; Hutt, N. M.; Environmental Geochemistry of Minesite Drainage: Practical and Case Studies, MDAG Publising, 1997.

10. Hammarstrom, J. M.; Kotlyar, B. B.; Theodore, T. G. Em Preliminary compilation of descriptive geoenvironmental mineral deposits models; Du Bray, E. A., ed.; U.S. Department of the Interior Open-File Report 95-831; Denver: USA, 1995, p. 90.

11. Kwong, Y. T. J.; MEND Project 1.32.1, Ottawa, Ontario: Canada, 1993, p. 47.

12. Taylor, C. D.; Zierenberg, R. A.; Goldfarb, R. J.; Sea, K. V.; Kleinkopf, M.D.; Em ref. 10, p. 137.

13. Cruz, R.; Bertrand, V.; Monroy, M.; González, I.; Appl. Geochem. 2001, 16,803 .

14. Doyle, F. M.; Mirza, A. H.; Proc. 4th International Symposium on Electrochemistry in Mineral and Metal Processing; Woods, R.; Doyle, F.M.; Richardson, P., eds.; The Electrochemical Society; USA, 1996, p. 203.

15. Nicholson, R. V.; Gillham, R. W.; Reardon, E. J.; Geochim. Cosmochim. Acta 1990, 54, 395.

16. Giannetti, G. F.; Rabockai, T.; J. Braz. Chem. Soc. 1993, 4, 61.

17. Jambor, J. L. Em Environmental Geochemistry of Sulfide Mine-Wastes; Jambor, J. L.; Blowes, W., eds.; Mineralogical Association of Canada, Canada, 1994, p. 59.

18. Cruz, R.; Monroy, M.; González, I.; Appl. Geochem. 2005, 20, 109.

19. Cruz, R.; Mendez, B. A.; Monroy, M.; González, I.; Appl. Geochem. 2001, $16,1631$. 